EDUCATION

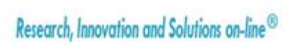

Electronic Journal of Research

in Educational Psychology

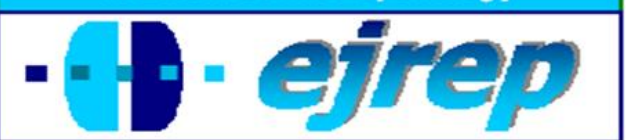

\title{
Actitudes hacia la Discapacidad en el Ámbito Educativo a través del SSCI (2000-2011). Análisis Temático y Bibliométrico
}

\section{José Manuel García-Fernández ${ }^{1}$, Cándido J. Inglés ${ }^{2}$, Maria Vicent Juan ${ }^{1}$, Carolina Gonzálvez Macià ${ }^{1}$ y Carmen Mañas Viejo}

${ }^{1}$ Psicología Evolutiva y Didáctica, Universidad de Alicante, Alicante. ${ }^{2}$ Psicología de la Salud, Universidad Miguel Hernández, Elche.

España

Correspondencia: JoséManuel García-Fernández.Campus de Sant Vicent del Raspeig. Ap. 99. E-03080. Alicante. España. E-mail: josemagf@ua.es

(c) Education \& Psychology I+D+i and Editorial EOS (Spain) 


\section{Resumen}

Introducción. El objetivo de este estudio es describir y caracterizar la producción científica a nivel internacional en relación al tópico "actitudes hacia la discapacidad en el ámbito educativo", a partir del uso de una batería de indicadores bibliométricos que permiten realizar un análisis y seguimiento de la actividad científica internacional.

Método. En este estudio ex post facto retrospectivo, se analizaron 925 documentos publicados en la base de datos Social Sciences Citation Index (SSCI) durante el periodo 2000-2011.

Resultados. El número de publicaciones creció desde 2006 a 2010 de forma progresiva. En el trabajo se indican los autores más productivos, las revistas especializadas en dicho tema y los artículos más citados, entre otros aspectos. El análisis según la tipología de la investigación mostró una tendencia a efectuar estudios empíricos. Al examinar el contenido de los artículos se identificaron 18 categorías temáticas así como un desequilibro en la clasificación de las investigaciones según el nivel educativo en el que se llevan a cabo.

Discusión y conclusiones. Los resultados obtenidos muestran la relevancia actual del tópico para la comunidad científica y nos ofrecen una visión sobre los rasgos que han caracterizado la investigación en materia de "actitudes hacia la discapacidad en el ámbito educativo" en los últimos once años.

Palabras Clave: Actitudes, discapacidad, educación, análisis bibliométrico. 


\title{
Attitudes towards Disability in Education through the SSCI (2000-2011): A Topical and Bibliometric Analysis
}

\begin{abstract}
Introduction. The purpose of this study is to describe the studies about "the attitudes towards disability in education" from the use of a battery of bibliometric indicators that allow monitoring and analysis of international scientific activity.
\end{abstract}

Method. This study ex post facto retrospective analyzed 925 papers published in 2000-2011 periods from the Social Sciences Citation Index (SSCI) database.

Results. The number of publications has increased from 2006 to 2010. The work shows firstly, the most productive authors. Secondly, the journals related to this topic, and finally, the most cited articles among other issues. In considering the content of the articles, there were identified 18 categories and an imbalance in the classification of researches depending on the educational level in which they are carried out.

Discussion and Conclusion. The results present the actual relevance of the topic for the scientific community and offer us a vision of the traits that have characterized research about "attitudes towards disability in education" for the last eleven years.

Keywords: Attitude, disability, education, bibliometric analysis.

Received: $12 / 15 / 12$

Initial acceptance: 02/10/13

Final acceptance: 03/08/13 


\section{Introducción}

Según el último Informe Mundial sobre la Discapacidad, elaborado por la OMS en 2011, se estima que más de mil millones de personas en todo el mundo viven con algún tipo de discapacidad, es decir, alrededor del $15 \%$ de la población mundial. El informe advierte que se trata de un problema social de gran relevancia, pues su prevalencia va en aumento, y a pesar de los múltiples esfuerzos y avances en materia de integración y mejora de la calidad de vida aun existe un gran número de obstáculos a los que se enfrentan las personas con discapacidad. Las actitudes negativas, constituyen una de las principales barreras para la integración de las personas discapacitadas en diversos sectores incluso cuando se han emprendido medidas legales para favorecer su integración (López-González y López-González, 1997).

Las actitudes han sido definidas desde el punto de vista psicológico por diversos autores como Ovejero (1998), Verdugo, Jenaro y Arias (1995) y, posteriormente, por GarcíaFernández, Good y Condés (2002). Para estos últimos "una actitud es una respuesta compleja y estable de un sujeto que supone la evaluación de un objeto, persona, grupo o entidad abstracta y que consta de aspectos cognitivos, afectivos y conductuales" (p.135).

En el marco de la educación inclusiva, el estudio y análisis de las actitudes hacia la discapacidad cobra especial relevancia, pues diversas investigaciones (Aguado, Flórez, y Alcedo, 2003, 2004; Almazán, 2003; González, 2008; Hughes et al., 2001; Krahé y Altwasser, 2006; Manetti, Schneider, y Siperstein, 2001; Piercy, Wilton, y Townsend, 2002; Shelvin y O’Moore, 2000) advierten sobre la persistencia de determinados prejuicios y creencias negativas en el ámbito educativo que dan lugar a actitudes de rechazo hacia los alumnos con discapacidad lo cual podría explicar el hecho de que, tal y como señalan Hogan, McLellan y Bauman (2000), Llewellyn (2000) y Piel (2007), la inclusión educativa de las personas con discapacidad no está produciendo los resultados positivos que se esperaban, pues el éxito de dicha inclusión depende en gran parte de las actitudes de los profesores y de los iguales, incluso cuando se posee un curriculum bien adaptado (Bueno, 2010; Garaigordobil y García de Galdeano, 2006; Jarvis y French, 1990; Larrivee y Cook, 1979; Navas, Torregrosa, y Mula, 2004; Rillota y Nettelbeck, 2007; Verdugo, Arias, y Jenaro, 1994). 
Actitudes de los profesionales de la Educación

La importancia de las actitudes que adopta el profesorado queda manifiesta por la gran cantidad de trabajos publicados en los últimos años centrados en el análisis de las actitudes docentes tanto hacia la integración e inclusión de las personas con discapacidad en los centros ordinarios (Boer, Pijl, y Minnaert, 2011; Cagran y Schmidt, 2011; Cook y Cameron, 2010; Cook, Tankersley, Cook, y Landrum, 2000; Finke, McNaughton, y Drager, 2009; Jordan, Glenn, y McGhie-Richmond, 2010; Kim, 2011; López, Echeita, y Martin, 2009; Moberg, 2003; Ocloo y Subbey, 2008; Parasuram, 2006) como hacia los alumnos con necesidades educativas especiales (Busch, Pederson, Espin, y Weissenburger, 2001; Kozub y Lienert, 2003; Murray, Lombardi, Wren, y Keys, 2009; Stephens, Stephens, y Von Eisenhart-Rothe, 2000; Woolfson y Brady, 2009). Esta doble vía de investigación se justifica por el hecho de que tal y como defiende Cochran (1997), las actitudes que se adoptan hacia la integración escolar reflejan, en gran medida, las actitudes hacia las personas discapacitadas en general.

Entre las variables relacionados con las actitudes que adoptan los profesores hacia los alumnos discapacitados en general, diversos estudios coinciden en que existe una cierta tendencia entre los profesores a manifestar actitudes más favorables cuanto más bajo es el nivel educativo al que atienden (Cochran, 1997; García y Alonso, 1985; Rogers, 1987). Por otro lado, se ha demostrado que los profesores aceptan mejor a los alumnos con discapacidades físicas que psíquicas (Cagran y Schmidt, 2011; Huang y Diamond, 2009; Soodak, Podell, y Lehman, 1998). En este sentido, Aguilera et al. (1990) advierten que aquello que más condiciona las actitudes, no es tanto el tipo de déficit sino el nivel intelectual asociado al déficit. Igualmente, las características personales y las conductas perjudiciales o provocadoras del propio discapacitado tienen una gran influencia en la adopción de actitudes negativas (Verdugo et al., 1995).

En relación a la opinión que tienen los profesores respecto a la integración de las personas con discapacidad, Alemany y Villuendas (2004) resumen las conclusiones obtenidas en los trabajos realizados por Mula et al. (2002) según las cuales la mayor parte de los docentes opinan que la formación en necesidades educativas especiales concierne a todo el profesorado y que la integración escolar favorece la preparación de los alumnos para vivir en una sociedad integrada. No obstante, un amplio sector de los docentes percibe que el proceso de integración ha sido más impuesto que consultado. Por otro lado, la mayoría de los profesores están de 
acuerdo en que la integración es una práctica educativa deseable aunque requiere un tiempo extra para atender a los alumnos con necesidades educativas especiales lo cual va en detrimento del resto de compañeros. Sin embargo, los estudios realizados por Blecker y Boakes (2010), revelan que los profesores en general, opinan que los alumnos se benefician de la interacción con sus compañeros sin discapacidad.

En cuanto a la formación del profesorado en materia de inclusión y atención a la diversidad, es indiscutible pensar que una adecuada formación que proporcione al profesorado la información y las herramientas necesarias y lo capacite para atender a los alumnos con discapacidad necesariamente propiciará la adopción de unas actitudes positivas tanto hacia la inclusión de dichos alumnos como hacia las personas con discapacidad en general. En este sentido, Martín-Domínguez y Soto-Rosales (2001) destacan la importancia más que de la formación, del sentimiento de competencia profesional para el trabajo con niños de necesidades educativas especiales, como factor determinante de actitudes. En cualquier caso, es necesario que, tanto desde los programas de formación de profesorado como desde las propias universidades, se lleve a cabo una apropiada preparación, tanto en lo personal-cultural, como en lo académico-profesional (Luque-Parra y Luque-Rojas, 2011).

\section{Actitudes del grupo de iguales}

Tal y como señalan Flórez, Aguado y Alcedo (2009, p.85) "las actitudes de los compañeros y pares de las personas con discapacidad tienen un efecto importante no sólo en el desarrollo del autoconcepto del propio sujeto con discapacidad, sino también en su proceso de socialización". En cuanto a los factores asociados con la adopción de actitudes más o menos favorables por parte del grupo de iguales, parece ser, que al igual que sucede con las actitudes del personal docente, las actitudes de los alumnos suelen ser más positivas ante estudiantes con discapacidades leves que severas (Rizzo y Vispoel, 1991) y ante discapacidades físicas que psíquicas (Brown, Ouellette-Kuntz, Lysaght y Burge, 2011). Del mismo modo, diversas investigaciones advierten que en general, las estudiantes suelen tener comportamientos más tolerantes hacia las personas con discapacidad que sus compañeros de sexo masculino (Litvack, Ritchie, y Shore, 2011; Slininger, Sherrill, y Jankowski, 2000; Vignes et al., 2009). Además, se considera que las actitudes de los iguales podrían verse influenciadas por factores como la edad, el nivel educativo y la etapa de formación. Sin embargo, se han enconrado resultados contradictorios para la variable edad, de manera que mientras las investigaciones 
realizadas con estudiantes universitarios encontraron que los estudiantes de los últimos cursos presentaron actitudes más positivas hacia la diversidad (Alonso, Navarro, y Vicente, 2008; Infante y Gómez, 2004), otras investigaciones realizadas en la etapa de educación secundaria encontraron actitudes más negativas en los cursos superiores que en los inferiores (Suriá, 2011). En este sentido, Livneh (1991), defiende que las actitudes hacia las personas con discapacidad van mejorando paulatinamente a través de las diversas etapas, lo cual explicaría los resultados obtenidos por las investigaciones llevadas a cabo por Gughwan y Chow (2001), según las cuales las actitudes de los estudiantes universitarios son más favorables que las de los alumnos de pregrado. Otra característica que parece tener influencia en el tipo de actitud adoptada es el nivel socioeconómico, puesto que a medida que este aumenta también aumenta la aceptación de la discapacidad (García-Fernández et al., 2002). Igualmente, el tener una buena calidad de vida es uno de los factores asociados a las actitudes positivas (Vignes et al., 2009).

Otro factor que parece influir en las actitudes que se adoptan hacia la discapacidad es el hecho de que los alumnos hayan tenido experiencias de convivencia directa con las personas con discapacidad (Suriá, 2011; Vignes et al., 2009). Estos resultados coinciden con las investigaciones realizadas por Flórez et al. (2009) que, tras realizar un análisis de los programas de cambio de actitudes realizados en el ámbito educativo desde 1997 hasta 2009, concluyeron que la técnica que mejor resultados reportaba era la del contacto directo con personas con discapacidad, siempre que dicho contacto implique un conocimiento mayor de la discapacidad.

Dada la relevancia actual del fenómeno de las actitudes hacia la discapacidad y el interés que ha suscitado desde la investigación, nos hemos propuesto realizar un análisis temático y bibliométrico sobre la documentación científica publicada en el SSCI desde el año 2000 hasta el 2011, con el objetivo de conocer la evolución y el estado actual de la investigación en "actitudes hacia la discapacidad en el ámbito educativo" mediante la evolución de la productividad, la productividad según la revista, la producción según el autor y el grado de colaboración entre autores, la cobertura temática identificada, la tipología de investigación y el número de citas que recibe cada documento. 


\section{Método}

\section{Muestra}

Se analizaron 925 documentos publicados en la base de datos Social Sciences Citation Index desde el año 2000 hasta el 2011.

\section{Materiales}

El material que se utilizó para esta investigación fue el Social Sciences Citation Index (SSCI) del ISI Web of Kowledge. El ISI Web of Kowledge es una plataforma que ofrece la posibilidad de realizar búsquedas a todo el contenido de las bases de datos multidisciplinares del Institut of Scientific Information: artículos de revistas, patentes, actas de congresos, herramientas de evaluación y análisis de la información publicada, tales como factor de impacto, índice de inmediatez, recursos de gestión bibliográfica y recursos Web.

El SSCI es una base de datos de revistas catalogadas como de mayor factor de impacto en el campo de las ciencias sociales y que se hace disponible en línea a través de la Web of Kowledge.

\section{Procedimiento}

Primeramente, se llevó a cabo la búsqueda de las unidades de análisis. Una vez seleccionadas las palabras clave de la búsqueda y tras varias combinaciones entre estas, se introdujo en la base de datos SSCI la estrategia de búsqueda escogida (attitude* and disabled or disabilit* and education) y se acotó al período comprendido entre el 2000 y el 2011, ambos incluidos. Aplicando esta estrategia se obtuvieron un total de 6.150 resultados. En cuanto a los criterios de exclusión, se descartaron los documentos que no mostraban resumen ni permitían acceder a su contenido completo, puesto que la información que nos ofrecen el título y las palabras clave es a menudo limitada y no nos permite conocer con exactitud el contenido del artículo. Una vez descartados estos documentos procedimos a la lectura de todos los resúmenes para asegurarnos de que su contenido se correspondía con nuestro tópico de investigación. En el caso de que el resumen fuese demasiado escueto o bien no aclarara con exactitud el contenido del documento realizamos la lectura del texto completo del documento. Tras esta rigurosa depuración, únicamente fueron seleccionados 925 documentos, los cuales conforman nuestra muestra. 
Una vez localizados físicamente los documentos se procedió a su lectura y a la selección de los datos necesarios para el posterior análisis siguiendo los indicadores bibliométricos que la propia base de datos SSCI nos proporcionó. Seguidamente se procedió a introducir los datos en el programa informático SPSS 18, para su posterior análisis estadístico, atendiendo a las siguientes variables: el "año de publicación", referida al año en que han sido publicados los artículos seleccionados, la variable "revista", que nos permite conocer el nombre de la revista en que cada artículo ha sido publicado y la variable "autores" referida al nombre de los autores y al número de firmas que recibe cada documento. La variable "categoría temática" subclasifica los documentos seleccionados en áreas temáticas relacionadas con el tema principal de nuestro estudio. El proceso de categorización se realizó tras una lectura global de los documentos, configurándose 18 categorías, posteriormente se volvieron a leer todos los documentos y a clasificar uno a uno en las categorías establecidas: Actitudes del personal docente (percepciones, opiniones, prejuicios y actitudes de los profesionales de la educación de los diversas niveles educativos); Actitudes del personal no docente (percepciones, opiniones, prejuicios y actitudes de los psicólogos, cuidadores, funcionarios, administradores, directores y terapeutas); Actitudes de los iguales (percepciones, opiniones, prejuicios y actitudes de los alumnos sin discapacidad); Actitudes sociales hacía la educación (percepciones, opiniones y actitudes de la sociedad hacia la educación de las personas discapacitadas); Prácticas de transición (barreras o dificultades y apoyos que reciben los alumnos con discapacidad durante las transiciones entre los distintos niveles educativos); Familia y escuela (refleja las actitudes como consecuencia de las barreras o dificultades y prácticas de apoyos y colaboración desde el ámbito educativo, así como la percepción de los familiares de los alumnos con discapacidad de dichas acciones); El Nivel Educativo nos indica si la investigación se ha desarrollado en el ámbito de la Educación Post Obligatoria (Educación Universitaria, Bachillerato y Formación Profesional), Educación Obligatoria (los diversos niveles que componen la educación obligatoria) o Educación Infantil (Atención Temprana, jardín de infancia y preescolar). A su vez, la categoría Educación Post Obligatoria, se subclasifica en Profesiones relacionadas con la Salud (Logopedia, Enfermería, Medicina...), Profesiones relacionadas con la Educación y Otras profesiones (Turismo, Psicología, Ciencias Sociales, Terapia Ocupacional...); Educación Física (posibilidades de interacción social e inclusión/segregación); Prácticas de Inclusión (programas de cambio de actitudes y prácticas para mejorar la inclusión y la interacción social de los alumnos no discapacitados con sus iguales); Relaciones Sociales de los ANEE con sus iguales (amistad, participación, interacción...); Políticas Educativas (políticas estatales, internacionales y de los propios centros educativos en relación a la educación de las 
personas discapacitadas); Evolución conceptual (análisis de las actitudes implícitas en las definiciones y conceptos referidos a la discapacidad en el contexto educativo); TIC y ANEE (brecha digital y posibilidades de participación/exclusión en el uso de las nuevas tecnologías para los estudiantes con discapacidad); Actitudes de las personas con discapacidad (percepciones y opiniones propias acerca de su educación), DPD (Desarrollo Profesional Docente: implicaciones para la formación del profesorado en materia de actitudes y atención de los alumnos con necesidades educativas especiales); Escuela Inclusiva (ventajas y desventajas, dilemas y controversias); Validación de Cuestionarios sobre actitudes hacia la discapacidad y Educación Sexual y discapacidad (actitudes sociales hacia la sexualidad de las personas con discapacidad e implicaciones para la educación).

Atendiendo al "tipo de investigación" se clasificaron los documentos según se tratasen de un informe de investigación empírico o teórico. Se consideró como trabajo teórico "todo trabajo en el que no se aporten datos empíricos originales de autores; es decir, todos los trabajos de revisión que no son un informe de una investigación empírica” (Montero y León, 2005, p.118). Otro parámetro considerado fue: el "número de citas", que hace referencia a las veces que ha sido citado cada documento hasta diciembre de 2011 según la Web of Science.

\section{Diseño y Análisis Estadísticos}

Este estudio historiográfico bibliométrico de los documentos seleccionados se realizó a través de un diseño ex post facto retrospectivo (Montero y León, 2005). Se utilizaron análisis descriptivos y de frecuencias para analizar las distintas variables. Todos los cálculos estadísticos se realizaron con el programa SPSS 18.

\section{Resultados}

Año de publicación

En cuanto a la distribución temporal de los 925 documentos, sobre nuestro tópico de estudio, publicados en el SSCI en los últimos once años (véase la Tabla 1), el rango de publicaciones por año oscila entre $46(5 \%)$ y $152(16,4 \%)$ documentos, siendo el promedio de $84,09(9,09 \%)$ documentos por año. La máxima cifra se alcanza durante el año 2010, mientras que durante los años 2003 y 2004 se produce el menor número de publicaciones. Desde el 
año 2000 se puede observar una cantidad considerable de publicaciones, produciéndose un ligero aumento al año siguiente, seguido de un leve descenso y una etapa en la que el número de publicaciones parece mantenerse estable durante cuatro años. Después de esta etapa de estabilidad, se produce un ligero incremento que comienza en el 2007, seguido de un gran aumento en las publicaciones al año siguiente llegando casi a doblar la producción del año anterior. En el año 2010 se alcanza el máximo número de publicaciones y en el 2011 se produce un gran descenso debido, probablemente, a la tardanza en la incorporación en la base de datos de los documentos publicados en este último año.

Tabla 1. Evolución temporal de la productividad

\begin{tabular}{ccc}
\hline Año & $\mathrm{n}$ & $(\%)$ \\
\hline 2000 & 48 & 5,2 \\
2001 & 64 & 6,9 \\
2002 & 53 & 5,7 \\
2003 & 46 & 5,0 \\
2004 & 46 & 5,0 \\
2005 & 47 & 5,1 \\
2006 & 47 & 5,1 \\
2007 & 68 & 7,4 \\
2008 & 124 & 13,4 \\
2009 & 150 & 16,2 \\
2010 & 152 & 16,4 \\
2011 & 80 & 8,6 \\
Total & 925 & 100 \\
\hline
\end{tabular}

\section{Revista}

Los documentos selecionados fueron publicados en un total de 270 revistas. En la Figura 1, se muestra una clasificación de las revistas según el número de documentos que han publicado sobre nuestro tópico de estudio.

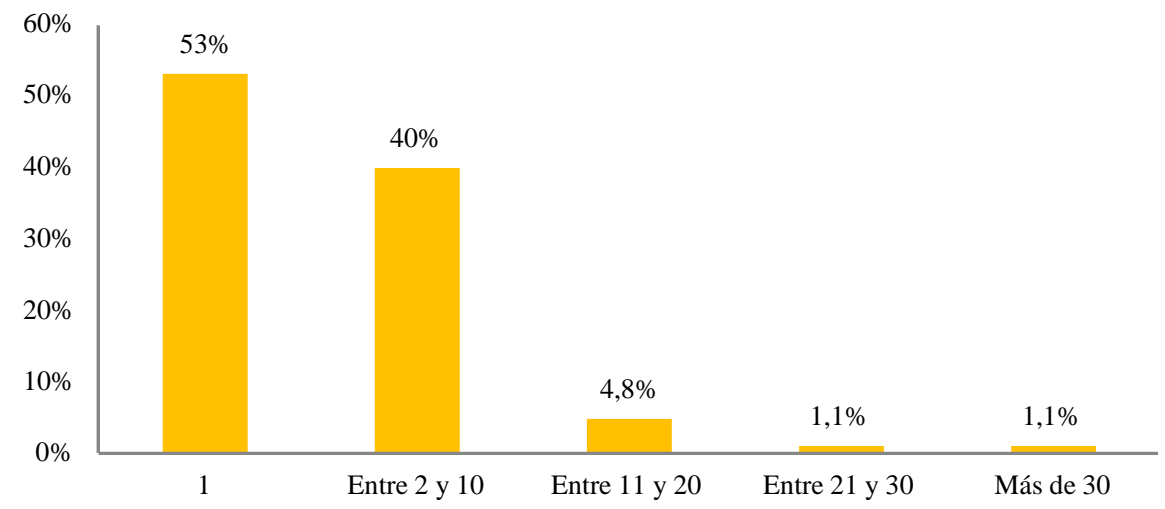

Figura 1. Clasificación de las revistas según el número de documentos que publican. 
Tal y como se observa, más de la mitad del total de revistas se compone por aquellas que únicamente han publicado un artículo sobre el tópico que se investiga, seguidas por aquellas que contienen entre dos y diez publicaciones, por lo que únicamente un $7 \%$ del total de las revistas analizadas ha publicado más de diez artículos durante el período acotado y, por tanto, pueden considerarse especializadas en nuestro tópico de investigación. En la Tabla 2 se han incluido todas aquellas revistas que han publicado más de diez artículos sobre nuestro tópico de investigación en los últimos once años, cuya producción supone un 40,5\% del total. Remedial and Special Education es la revista con mayor número de publicaciones sobre el tema de este estudio, con un total de 52 artículos, seguida de Disability \& Society con 43 publicaciones y por International Journal of Inclusive Education con 33 documentos publicados.

Tabla 2. Producción de las revistas

\begin{tabular}{lcc}
\hline \multicolumn{1}{c}{ Revista } & $\mathrm{n}$ & $(\%)$ \\
\hline - REMEDIAL AND SPECIAL EDUCATION & 52 & 5,6 \\
- DISABILITY \& SOCIETY & 43 & 4,6 \\
- INTERNATIONAL JOURNAL OF INCLUSIVE EDUCA- & & 3,6 \\
TION & 33 & 3,2 \\
- EXCEPTIONAL CHILDREN & 30 & 3,1 \\
- ACADEMIC MEDICINE & 29 & 2,8 \\
- RESEARCH AND PRACTICE FOR PERSONS WITH SE- & 26 & 1,9 \\
VERE DISABILITIES & 18 & 1,7 \\
- JOURNAL OF SPECIAL EDUCATION & 16 & 1,7 \\
- INTERVENTION IN SCHOOL AND CLINIC & 16 & 1,6 \\
- TOPICS IN EARLY CHILDHOOD SPECIAL EDUCATION & & 1,6 \\
- EDUCATION AND TRAINING IN DEVELOPMENTAL & 15 & 1,5 \\
DISABILITIES & 15 & 1,4 \\
- LEARNING DISABILITY QUARTERLY & & 1,4 \\
- INTERNATIONAL JOURNAL OF DISABILITY DEVEL- & 14 & \\
OPMENT AND EDUCATION & 13 & 1,2 \\
- TEACHING AND TEACHER EDUCATION & & \\
- JOURNAL OF INTELLECTUAL DISABILITY RE- & 13 & 1,2 \\
SEARCH & & \\
- JOURNAL OF APPLIED RESEARCH IN INTELLECTUAL & 11 & 1,2 \\
DISABILITIES & & 1,2 \\
- JOURNAL OF DEVELOPMENTAL AND PHYSICAL & 11 & \\
DISABILITIES & & \\
- JOURNAL OF POLICY AND PRACTICE IN INTELLEC- & 11 & \\
TUAL DISABILITIES & 11 & \\
- RESEARCH QUARTERLY FOR EXERCISE AND SPORT & 11 & \\
TOtal & & \\
\hline
\end{tabular}




\section{Autores}

Teniendo en cuenta la clasificación propuesta por Crane (1969) podemos establecer cuatro categorías de autores según su productividad: grandes productores (10 o más trabajos), productores moderados (5-9 trabajos), aspirantes (2-4 trabajos) y transeúntes (1 trabajo). Se han identificado un total de 1882 autores, de los cuales, un único autor se ha considerado como gran productor, suponiento el 0,05 de la muestra. En el caso de los productores moderados, 15 son los autores y representan el $0,80 \%$ de la muestra; los aspirantes son 280 y suponen el 14, 88\%, mientras que 1586 autores se han considerado transeúntes, representando el 84, 27\% restante (véase la figura 2). En la Tabla 3 se recoge el listado de los autores moderados y grandes productores. En lo que respecta a los autores españoles, destacan Alcedo-Rodríguez, M.A., Aguado-Díaz, A. y Echeita, G. con dos publicaciones, cada uno, sobre nuestro tópico de estudio.

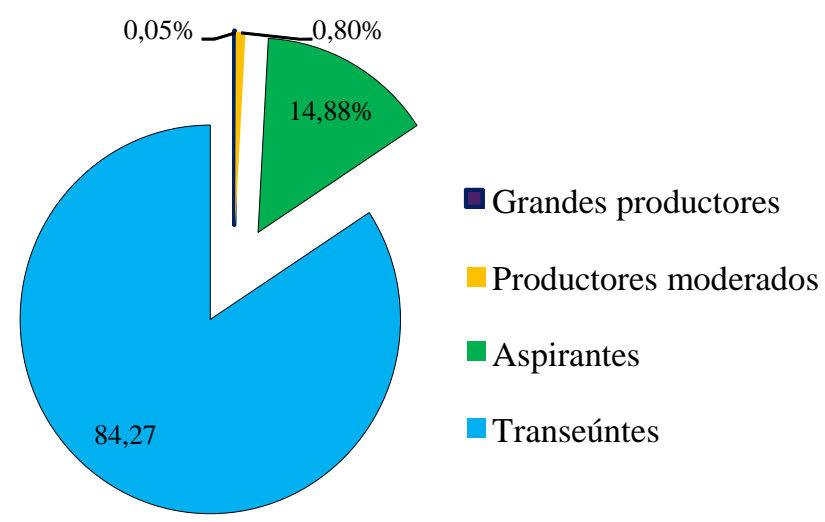

Figura 2. Clasificación de los autores según su productividad.

Tabla 3. Autores más productivos durante el período 2000-2011

\begin{tabular}{lcll}
\hline Autor & $\mathrm{n}$ & Autor & $\mathrm{n}$ \\
\hline Carter, E. & 10 & Copeland, S.R. & 7 \\
Hughes, C. & 9 & Kauffman, J.M. & 7 \\
Wehmeyer, M. & 9 & Obrusnikova, I. & 7 \\
Chan, F. & 8 & Agram, M. & 6 \\
Forlin, C. & 8 & Downing, J. & 5 \\
Hodge, S. & 8 & Pijl, S.J. & 5 \\
Turnbull, A. & 8 & Sharma, U. & 5 \\
Bolck, M. & 7 & Van Hove, G. & 5 \\
\hline
\end{tabular}


El rango de número de firmas de autor por documento oscila entre una y once. El índice de coautoría o número de autores promedio es de 2,55 por trabajo. En la evaluación se hallaron 668 documentos escritos por dos o más autores (véase la Tabla 4) por lo que la colaboración asciende al 72,2\% del total de la muestra, siendo los trabajos firmados por dos autores los más numerosos $(30,1 \%)$.

\begin{tabular}{lcc}
\multicolumn{3}{c}{ Tabla 4. Colaboración entre autores } \\
\hline $\mathrm{N}^{\mathrm{o}}$ de colaboradores & $\mathrm{n}$ & $(\%)$ \\
\hline 1 & 257 & 27,8 \\
2 & 278 & 30,1 \\
3 & 187 & 20,2 \\
4 & 103 & 11,1 \\
5 & 49 & 5,3 \\
6 & 30 & 3,2 \\
7 & 16 & 1,7 \\
8 & 2 & 0,2 \\
11 & 2 & 0,2 \\
Inespecífico & 1 & 0,1 \\
Total & 925 & 100 \\
\hline
\end{tabular}

\section{Categoría temática}

Tras un análisis detenido del contenido de los 925 artículos que conforman la muestra seleccionada, se identificaron 18 categorías temáticas en relación a nuestro tópico de investigación. Dichas temáticas no son exluyentes unas de otras, de manera que un mismo artículo pudo ser clasificado en más de una categoría temática. Dicha clasificación, como puede observarse en la Tabla 5, pone de manifiesto que la categoría sobre la que más se publica es Actitudes del personal docente con una presencia del $12,2 \%$ en el total de la muestra, seguida por la temática Prácticas de inclusión presente en un 9,9\% de los documentos seleccionados y, en tercer lugar, la temática Familia y escuela, con un 9,6\% de presencia en el total de la muestra. 
Tabla 5. Productividad según la categoría temática

\begin{tabular}{lcclcc}
\hline Categorías Temáticas & $\mathrm{n}$ & $(\%)$ & Categorías Temáticas & $\mathrm{n}$ & $(\%)$ \\
\hline $\begin{array}{l}\text { Actitudes del personal } \\
\text { docente }\end{array}$ & 113 & 12,2 & Actitudes sociales & 32 & 3,5 \\
$\begin{array}{l}\text { Prácticas de inclusión } \\
\text { Familia y escuela }\end{array}$ & 92 & 9,9 & $\begin{array}{l}\text { Escuela inclusiva } \\
\text { Actitudes del perso- }\end{array}$ & 30 & 3,2 \\
nolíticas educativas & 76 & 8,2 & $\begin{array}{l}\text { Validación de cues- } \\
\text { tionarios }\end{array}$ & 24 & 2,6 \\
Educación física & 63 & 6,8 & $\begin{array}{l}\text { Prácticas de transi- } \\
\text { ción }\end{array}$ & 21 & 2,3 \\
$\begin{array}{l}\text { DPD } \\
\text { Relaciones sociales de } \\
\text { los ANEE con sus }\end{array}$ & 56 & 6,1 & $\begin{array}{l}\text { TIC y ANEE } \\
\text { Educación sexual y }\end{array}$ & 20 & 2,2 \\
$\begin{array}{l}\text { iguales } \\
\text { Actitudes de los igua- }\end{array}$ & 41 & 4,4 & Evolución conceptual & 17 & 1,8 \\
$\begin{array}{l}\text { les } \\
\text { Actitudes de las per- } \\
\text { sonas con discapaci- } \\
\text { dad }\end{array}$ & 36 & 3,9 & & & 1,6 \\
\hline
\end{tabular}

Unicamente se identificó el nivel educativo en el que se centraba la investigación en 329 artículos. Tal y como muestra la Tabla 6, predominan las investigaciones realizadas en la Educación Obligatoria, mientras que los estudios realizados en la etapa Infantil constituyen el menor porcentaje, diferenciandose claramente de las etapas que la suceden.

Tabla 6. Clasificación de los estudios según el nivel educativo

\begin{tabular}{lcc}
\hline \multicolumn{1}{c}{ Nivel educativo } & $\mathrm{n}$ & $(\%)$ \\
\hline $\begin{array}{l}\text { Educación Post Obliga- } \\
\text { toria }\end{array}$ & 132 & 14,3 \\
Educación Obligatoria & 148 & 16 \\
Educación Infantil & 49 & 5,3 \\
Total & 329 & 35,6 \\
\hline
\end{tabular}

A su vez, la etapa de Educación Post Obligatoria se subclasifica según el área profesional en la que se centra la investigación, predominando las profesiones relacionadas con la salud (véase la Figura 3). 


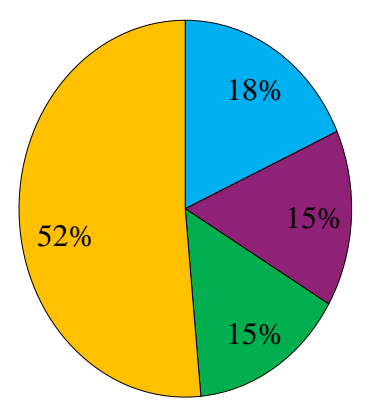

- Profesiones relacionadas con la salud

- Profesiones relacionadas con la educación

Otras profesiones

- Inespecífico

\section{Figura 3.Clasificación de las subcategorías pertenecientes a la temática Educación Post Obligatoria.}

Tipo de investigación

En cuanto al tipo de investigación, 773 documentos se han identificado como empíricos, lo cual supone la mayor parte de la producción $(83,6 \%)$. El 16, 4\% de la muestra restante se identificó como trabajos teóricos.

\section{Número de citas}

El total de citas que reciben los 925 documentos seleccionados hasta diciembre de 2011 según la Web of Science es de 4216 citas, con un rango entre 0 y 85, siendo el promedio de 4,56 por documento. La mayor parte de los documentos seleccionados $(55,2 \%)$ reciben entre una y 15 citas. Los trabajos que han recibido más de 45 citas, representan el 0,6\% del total de la muestra. El $37 \%$ de la muestra está constituido por aquellos trabajos que hasta la fecha consultada no habían recibido ninguna cita (véase la Figura 4).

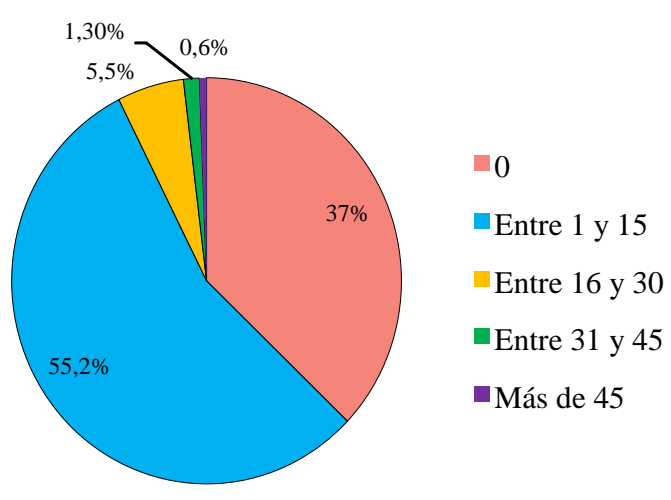

Figura 4 . Número de citas por documento. 
La Tabla 7 contiene todos los artículos que aparecen más de 30 veces citados en la Web of Science. La mayoría de los autores de los artículos más citados no se han clasificado anteriormente como productores moderados o grandes productores, a excepción de Wehmeyer, M. y Downing, J.E. Del mismo modo, de los 14 artículos incluidos en la tabla 7, solamente cinco pertenecen a revistas clasificadas anteriormente como especializadas.

Tabla 7. Artículos más citados

\begin{tabular}{ll}
\hline Artículo & $\mathrm{N}^{\mathbf{o}} \mathrm{de}$ \\
& $\mathrm{Citas}$ \\
\hline
\end{tabular}

- Pinfold, V., Toulmin, H., Thornicroft, G., Huxley, P., Farmer, P., y Graham, T. (2003). Reducing psychiatric stigma and discrimination: evaluation of educational interventions in UK secondary schools. British Journal of Psychiatry, 182, 342-346.

- Benz, M.R., Lindstrom, L., y Yovanoff, P. (2000). Improving graduation and employment outcomes of students with disabilities: Predictive factors and student perspectives. Exceptional Children, 66(4), 509-529.

- Kavale, K.A., y Forness, S.R. (2000). What definitions of learning disability say and don't say - A critical analysis. Journal of Learning Disabilities, 33(3), 239-256.

- Park, J.H., Faulkner, J., y Schaller, M. (2003). Evolved disease-avoidance processes and contemporary anti-social behavior: Prejudicial attitudes and avoidance of people with physical disabilities. Journal of Nonverbal Behavior, 27(2), 65-87.

- Antonak, R.F., y Livneh, H. (2000). Measurement of attitudes towards persons with disabilities. Disability and Rehabilitation, 22(5), 211-224.

- Corrigan, P., Thompson, V., Lambert, D., Sangster, Y., Noel, J.G., y Campbell, J. (2003). Perceptions of discrimination among persons with serious mental illness. Psychiatric Services, 54(8), 1105-1110.

- Kavale, K.A., y Forness, S.R. (2000). History, rhetoric, and reality - Analysis of the inclusion debate. Remedial and Special Education, 21(5), 279-296.

Citas

- Artiles, A.J. (2003). Special education's changing identity: Paradoxes and dilemmas in views of culture and space. Harvard Educational Review, 73(2), 164202.

- Odom, S.L. (2000). Preschool inclusion: What we know and where we go from here. Topics in Early Childhood Special Education, 20(1), 20-27.

- Agran, M., Alper, S., y Wehmeyer, M. (2002). Access to the general curriculum for students with significant disabilities: what it means to teachers. Education and Training in Mental Retardation and Developmental Disabilities, 37(2), 123133.

- Ricci, L.A., y Hodapp, R.M. (2003). Fathers of children with Down's syndrome versus other types of intellectual disability: perceptions, stress and involvement. Journal of Intellectual Disability Research, 47, 273-284.

- Goodwin, D.L., y Watkinson, E.J. (2000). Inclusive physical education from the perspective of students with physical disabilities. Adapted Physical Activity Quarterly, 17(2), 144-160.

- Downing, J.E., Ryndak, D.L., y Clark, D. (2000). Paraeducators in inclusive classrooms - Their own perceptions. Remedial and Special Education, 21(3), 171-181.

- Gostin, L.O. (2000). Human rights of persons with mental disabilities - The European Convention of Human Rights. International Journal of Law And Psychiatry, 23(2), 125-159. 


\section{Discusión y conclusiones}

El presente estudio bibliométrico ofrece una visión sobre la evolución y las perspectivas de los estudios sobre actitudes hacia la discapacidad en el ámbito educativo. En el período 2000-2011, la productividad sobre actitudes hacia la discapacidad en el ámbito educativo puede considerarse moderada, siendo la media de 84,09 documentos por año. No obstante, el incremento en el número de publicaciones a partir del año 2006 nos indica que se trata de un tópico de actual relevancia. Asimismo, tal y como se ha nombrado anteriormente, en el año 2011 se observa un descenso en el número de publicaciones sobre nuestro tópico de estudio. Este hecho se debe probablemente a la demora en la indexación en la base de datos SSCI de los documentos publicados en el último año. Este creciente interés de la comunidad científica podría estar motivado por el aumento de políticas educativas de tipo inclusivas y el interés de los gobiernos por garantizar la integración de las personas discapacitadas en el ámbito educativo, pues parece existir una cierta influencia entre las actitudes que se adoptan y el éxito de la inclusión educativa (Bueno, 2010; Garaigordobil y García de Galdeano, 2006; Jarvis y French, 1990; Navas et al., 2004; Rillota y Nettelbeck, 2007; Verdugo et al., 1994).

La concentración de un 40,5\% de los documentos en un número reducido de títulos de revistas, ofrece un perfil de las revistas especializadas y preferentes para publicar sobre el área y poder encontrar información específica sobre la misma. Destacan Remedial and Special Education, Disability \& Society e International Journal of Inclusive Education como las tres revistas más productivas, respectivamente, en relación con nuestro tema de estudio.

Los hallazgos acerca de la productividad de los autores indican un predominio de autores transeúntes, resultado habitual en los estudios bibliométricos (Quiles, Ortigosa, Pedroche, y Méndez, 2000). Así mismo, se identificó un único gran productor (Carter, E.) cuya firma aparece en diez de los documentos que conforman la muestra. En cuanto a la colaboración entre los investigadores, es una tendencia que ha aumentado en los últimos años, aunque algunos autores advierten que se ha producido de forma más lenta en el ámbito de las Ciencias Sociales (Aguado-López et al., 2009; Anta, 2008; De Filippo, Sanz-Casado y Gómez, 2007; Frame y Carpenter, 1979). Sin embargo, los resultados de nuestro estudio acerca del índice de colaboración entre autores nos indican una tendencia a efectuar el trabajo en colaboración, siendo el índice de coautoría de 2,55 autores por trabajo. Este resultado coincide con 
varios estudios bibliométricos recientes relacionados con el campo de la psicología cuyos índices de colaboración parecen indicar una disposición a efectuar los trabajos en equipos de 2 a 3 autores (Agudelo, Bretón-López, y Buela-Casal, 2003; Alcain y Carpintero, 2001; Gómez, Hidalgo, Guilera, y Moreno, 2005; Guilera, Gómez, y Hidalgo, 2006; MorenoRodríguez y Sánchez-Moreno, 1998; Osca-Lluch, Civera, y Tortosa, 2012). Probablemente las recientes políticas destinadas a fomentar la movilidad de personal, tanto a nivel nacional como internacional, han contribuido a reforzar los vínculos entre el personal de las distintas instituciones favoreciendo la creación de equipos de trabajo (Carayol y Matt, 2004; Kliegl y Bates, 2011).

En cuanto al análisis de contenido de los documentos, los resultados parecen indicar que la investigación en el campo de las actitudes hacia la discapacidad en el ámbito educativo es eminentemente práctica. El predominio de la investigaciones prácticas frente a las teóricas también caracteriza la investigación sobre otros tópicos de estudio en el campo de la psicología (Cantón-Chirivella y Domingo-Tarín, 2008). El análisis de las categorías temáticas manifiesta una gran diversidad de líneas de investigación, predominando el interés por las actitudes que adoptan los propios profesionales de la educación, así como las prácticas que pueden ayudar a mejorar la inclusión de los alumnos discapacitados y las relaciones de colaboración y apoyo que se establecen entre los familiares de los alumnos discapacitados y la escuela. Atendiendo al nivel educativo, destaca el predominio de las investigaciones llevadas a cabo en las distintas etapas de Educación Obligatoria seguidas por los estudios sobre Educación Post Obligatoria, también con una producción cuantitativamente aceptable. No obstante, parece que las actitudes hacia la discapacidad en la etapa de Educación Infantil no gozan de tanto interés por parte de los investigadores como las etapas posteriores, aspecto del que ya alertaron Flórez, et al. (2009) tras realizar un análisis de programas de cambio de actitudes hacia la discapacidad. Igualmente, parece que este hecho no sólo afecta al tópico de nuestra investigación, puesto que se han obtenido resultados similares en estudios bibliométricos sobre la investigación educativa en general (Anta, 2008).

La revisión presentada en este trabajo tiene varias limitaciones. Así, no se analizaron variables potencialmente interesantes como la procedencia geográfica y profesional y el sexo de los autores, y tampoco se tuvieron en cuenta aspectos relativos a la financiación de los trabajos publicados. Otra posible limitación es el hecho de que no se ha encontrado ningún estudio bibliométrico sobre nuestro tópico de investigación con el que puedan ser contrastados 
nuestros resultados. Por otro lado, nuestro estudio se centra únicamente en las actitudes adoptadas dentro del terreno educativo, por lo que creemos que una posible línea de investigación consistiría en realizar un estudio más amplio que abarcara diversos ámbitos sociales igualmente interesantes.

El campo educativo parece ser un terreno fértil para el estudio de las actitudes hacia las personas con discapacidad, prueba de ello es la gran cantidad de producción científica publicada durante los últimos años. El presente estudio bibliométrico nos permite conocer el estado de la cuestión sobre nuestro tema de estudio (Agudelo, Bretón-López, y Buela-Casal, 2004; García-Sílberman, Arana, Martínez, Infante, y Jiménez, 2004; Quevedo y López-López, 2010) puesto que ofrece una imagen representativa sobre cómo se ha investigado dicho tópico en los últimos once años, los procesos de comunicación, los cursos de desarrollo así como las tendencias que interesaría potenciar o corregir. Igualmente, este estudio puede facilitar la tarea de los investigadores que estén interesados en investigar acerca de las actitudes hacia la discapacidad en el ámbito educativo, ya que contiene información sobre los autores más productivos, las revistas especializadas, los artículos más citados y otras referencias que pueden ser de utilidad para futuras investigaciones. Así mismo, esperamos que nuestro trabajo estimule la realización de nuevas investigaciones sobre actitudes hacia la discapacidad en el ámbito educativo, un tema de vital importancia si queremos llevar a cabo de forma efectiva un proyecto de envergadura como es la inclusión educativa.

\section{Referencias}

Aguado, A.L., Flórez, M.A., y Alcedo, M.A. (2003). Un programa de cambio de actitudes hacia personas con discapacidad en entorno escolar. Análisis y Modificación de Conducta, 29(127), 673-704.

Aguado, A.L., Flórez, M.A., y Alcedo, M.A. (2004). Programas de cambio de actitudes ante la discapacidad. Psicothema, 16(4), 667-673.

Aguado-López, E., Rogel-Salazar, R., Garduño-Oropeza, G., Becerril-García, A., ZúñigaRoca, M.F., y Velázquez-Álvarez, A. (2009). Patrones de colaboración científica a partir de redes de coautoría. Convergencia, 16, 225-258.

Agudelo, D., Bretón-López, J., y Buela-Casal, G. (2003). Análisis bibliométrico de las revistas de Psicología clínica editadas en castellano. Psicothema, 15(4), 507-516. 
Agudelo, D., Bretón-López, J., y Buela-Casal, G. (2004). Análisis bibliométrico de las revistas relacionadas con psicología de la salud editadas en castellano. Salud mental, 27(2), $70-85$.

Aguilera, M.J., Alvarez, K., Babio, M., Coll, C., Echeita, G., Galan, M., Marchesi, A., Martín, E., y Martínez-Arias, R. (1990). Evaluación del programa de integración escolar de alumnos con deficiencias. Madrid: MEC-CIDE.

Alcain, M.D., y Carpintero, H. (2001). La Psicología en España a través de las revistas internacionales: 1981-1999. Papeles del Psicólogo, 78, 11-20.

Alemany, I., y Villuendas, M.D. (2004). Las actitudes del profesorado hacia el alumnado con necesidades educativas especiales. Convergencia. Revista de Ciencias Sociales, 11(034), 183-215.

Almazán, L. (2003). Los cambios actitudinales hacia la integración escolar desde la perspectiva de los alumnos. Revista Fuentes, 5, 135-152.

Alonso, Mª J., Navarro, R., y Vicente, L. (2008). Actitudes hacia la diversidad en estudiantes universitarios. Trabajo presentado en las XIII Jornadas de Fomento de la Investigación, Universitat Jaume I, Castellón.

Anta, C. (2008). Análisis bibliométrico de la investigación educativa divulgada en publicaciones periódicas españolas entre 1990-2002. Revista Electrónica de Investigación Educativa, 10(1). Recuperado de http://redie.uabc.mx/vol10no1/contenido-anta.html

Blecker, N.S., y Boakes, N.J. (2010). Creating a learning environment for all children: are teachers able and willing?. International Journal of Inclusive Education, 14(5), 435447.

Boer, A.A., Pijl, S.J., y Minnaert, A. (2011). Regular primary school teachers' attitudes towards inclusive education: A review of the literature. International Journal of Inclusive Education, 15(3), 331-353.

Brown, H.K., Ouellette-Kuntz, H., Lysaght, R., y Burge, P. (2011). Students' Behavioural Intentions Towards Peers with Disability. Journal of Applied Research in Intellectual Disabilities, 24(4), 322-332.

Bueno, A. (2010). Una mirada ilusionada al futuro de los Servicios de Apoyo a la discapacidad en la Universidad. Buenas prácticas de apoyo a la discapacidad en la universidad. En A. Bueno, Buenas prácticas durante los estudios universitarios en la recepción de la información (pp.10-13). Alicante: Editorial CEE Limencop. 
Busch, T.W., Pederson, K., Espin, C.A., y Weissenburger, J.W. (2001). Teaching students with learning disabilities: Perceptions of a first-year teacher. Journal of Special Education, 35(2), 92-99.

Cagran, B., y Schmidt, M. (2011). Attitudes of Slovene teachers towards the inclusion of pupils with different types of special needs in primary school. Educational Studies, 37(2), 171-195.

Cantón-Chirivella, E., y Domingo-Tarín, A. (2008). Diabetes insulinodependiente y depresión: Análisis de la investigación reciente. LIBERABIT, 14, 41-48.

Carayol, N., y Matt, M. (2004). Does research organization influence academic production?. Research Policy, 33(8), 1081-1102.

Carvalho-Freitas, M. N., Leal, G. T., y Souto, J. F. (2011). Disability and Work: International Scientific Literature. Pesquisas e Práticas Psicossociais, 6(1), 128-138.

Cochran, H. (1997). The development and psychometric analysis of the scale of teachers' attitudes toward inclusion. Dissertation Abstracts International, 58(6A), 2068.

Cook, B.G., y Cameron, D.L. (2010). Inclusive Teachers' Concern and Rejection Toward Their Students Investigating the Validity of Ratings and Comparing Student Groups. Remedial and Special Education, 31(2), 67-76.

Cook, B.G., Tankersley, M., Cook, L., y Landrum, T.J. (2000). Teachers' attitudes toward their included students with disabilities. Exceptional Children, 67(1), 115-135.

Crane, D. (1969). Social Structures in a Group of Scientist: a test of the "Invisible College" Hypothesis. American Sociological Review, 34, 335-352.

De Filippo, D., Sanz-Casado, E., y Gómez, I. (2007). Movilidad de investigadores y producción en coautoría para el estudio de la colaboración científica. Revista CTS, 3(8), 23-40.

Finke, E.H., McNaughton, D.B., y Drager, K.D.R. (2009). All children can and should have the opportunity to learn: General education teachers' perspectives on including children with autism spectrum disorder who require AAC. Augmentative and Alternative Communication, 25(2), 110-122.

Flórez, M.A., Aguado, A.L., y Alcedo, M.A. (2009). Revisión y análisis de los programas de cambio de actitudes hacia personas con discapacidad. Anuario de Psicología Clínica y de la Salud, 5, 85-98.

Frame, J.D., y Carpenter, M.P. (1979). International research collaboration. Social Studies of Science, 9, 481-97.

Garaigordobil, M., y García de Galdeano, P. (2006). Empatía en niños de 10 a 12 años. Psicothema, 18(2), 180-186. 
García, J.N., y Alonso, J.C. (1985). Actitudes de los maestros hacia la integración escolar de niños con necesidades educativas especiales, Infancia y Aprendizaje, 30, 51-68.

García-Sílberman, S., Arana, D., Martínez, R., Infante, R., y Jiménez, A. (2004). La investigación sobre aspectos epidemiológicos y psicosociales de salud mental: Un análisis bibliométrico. Salud mental, 27(5) 8-22.

García-Fernández, J.M., Good, G.M., y Condés, N. (2002). Actitudes hacia la discapacidad. En J.M. García Fernández, J. Pérez Cobacho y P.P. Berruezo Adelantado (Eds.), Discapacidad Intelectual. Desarrollo, comunicación e intervención (pp.135-153). Madrid: Editorial CEPE.

Gómez, J., Hidalgo, M.D., Guilera, G., y Moreno, M. (2005). A bibliometric study of differential item functioning. Scientometrics, 64(1), 3-16.

González, R. (2008). Programas de cambio de actitudes hacia la discapacidad. Proyecto de investigación. Universidad de Oviedo. Xerocopiado.

Gughwan, C.H., y Chow, L. (2001). Korean students' differential attitudes toward people with disabilities: an acculturation perspective. International Journal of Rehabilitation Research, 24, 79-81.

Guilera, G., Gómez, J., y Hidalgo, D. (2006). Funcionamiento diferencial de los ítems: un análisis bibliométrico de las revistas editadas en español. Psicothema, 18(4), 841-847.

Hogan, A., McLellan, L., y Bauman, A. (2000). Health promotion needs of young people with disabilities: A population study. Disability and Rehabilitation, 22(8), 352-357.

Huang, H.H. y, Diamond, K.E. (2009). Early childhood teachers' ideas about including children with disabilities in programmes designed for typically developing children. International Journal of Disability, Development and Education, 56(2), 169-182.

Hughes, C., Copeland, S.R., Guth, C., Rung, L.L., Hwang, B., Kleeb, G., y Strong, M. (2001). General education students perspectives on their involvement in a High School peer buddy program. Education and Training in Mental Retardation and Developmental Disabilities, 36(4), 343-356.

Infante, M., y Gómez, V. (2004). Actitudes de los estudiantes de educación hacia la integración de personas con discapacidad y hacia la educación multicultural. Cultura y Educación, 16(4), 371-384.

Jarvis, K.C., y French, R. (1990). Attitudes of physical educators toward the integration of handicapped students. Perceptual and Motor Skills, 70, 899-902.

Jordan, A., Glenn, C., y McGhie-Richmond, D. (2010). The Supporting Effective Teaching (SET) project: The relationship of inclusive teaching practices to teachers' beliefs about 
disability and ability, and about their roles as teachers. Teaching and Teacher Education, 26(2), 259-266.

Kim, J.R. (2011). Influence of teacher preparation programmes on preservice teachers' attitudes toward inclusion. International Journal of Inclusive Education, 15(3), 355-377.

Kliegl, R., y Bates, D. (2011). International Collaboration in Psychology is on the Rise. Scientometrics, 87(1), 149-158.

Kozub, F.M., y Lienert, C. (2003). Attitudes toward teaching children with disabilities: A review of literature and research paradigm for future research. Adapted Physical Activity Quarterly, 20(4), 323-346.

Krahé, B., y Altwasser, C. (2006). Changing negative attitudes towards persons with physical disabilities: an experimental intervention. Journal of Community and Applied Social Psychology, 16(1), 59-69.

Larrivee, B., y Cook, L. (1979). Mainstreaming: a study of the variables affecting teacher attitude. The Journal of Special Education, 13(3), 315-324.

Litvack, M.S., Ritchie, K.C., y Shore, B.M. (2011). High- and average-achieving students' perceptions of disabilities and of students with disabilities in inclusive classrooms. $E x$ ceptional Children, 77(4), 474-487.

Livneh, H. (1991). On the origins of negative attitudes toward people with disabilities. En R.P. Marinelli, y A.E. Dell Orto (Eds.), The psychological and social impact of disability (pp.181-196). New York: Springer Publishing Company.

Llewellyn, A. (2000). Perceptions of mainstreaming: a systems approach. Developmental Medicine and Child Neurology, 42(2), 106-115.

López, M., Echeita, G., y Martín, E. (2009). Concepciones sobre el proceso de inclusión educativa de alumnos con discapacidad intelectual en la educación secundaria obligatoria. Cultura y Educación, 21(4), 485-496.

López-González, M., y López-González, M. (1997). Simular la discapacidad. Una técnica para conocer las necesidades educativas especiales y modificar actitudes en la formación del profesorado. Revista Electrónica Interuniversitaria de Formación del Profesorado, $1(0), 1-5$.

Luque-Parra, D., y Luque-Rojas, M.J. (2011). Conocimiento de la discapacidad y relaciones sociales en el aula inclusiva. Sugerencias para la acción tutorial. Revista Iberoamericana de Educación, 54(6), 1-12. 
Manetti, M., Schneider, B.H., y Siperstein, G. (2001). Social acceptance of children with mental retardation: Testing the contact hypothesis with an Italian sample. International Journal of Behavioral Development, 25(3), 279-286.

Martín-Domínguez, D., y Soto-Rosales, A. (2001). La atención a la diversidad. Una cuestión de actitudes. XXI, Revista de educación, 3, 149-157.

Moberg, S. (2003). Education for all in the North and the South: Teachers' Attitudes Towards Inclusive Education in Finland and Zambia. Education and Training in Developmental Disabilities, 38(4), 417-428.

Montero, I., y León, O.G. (2005). Sistema de clasificación del método en los informes de investigación en psicología. International Journal of Clinical and Health Psychology, 5(001), 115-127.

Moreno-Rodríguez, S., y Sánchez-Moreno, A. (1998). Análisis bibliométrico de la revista «Psicothema» (1989-1997). Psicothema, 10(1), 23-27.

Murray, C., Lombardi, A., Wren, C.T., y Keys, C. (2009). Associations between prior disability-focused training and disability-related attitudes and perceptions among university faculty. Learning Disability Quarterly, 32(2), 87-100.

Navas, L., Torregrosa, G., y Mula, A. (2004). Algunas variables predictoras de las actitudes del alumnado ante la integración escolar. Revista de Psicología Social, 19(2), 159-171.

Ocloo, M.A., y Subbey, M. (2008). Perception of basic education school teachers towards inclusive education in the Hohoe District of Ghana. International Journal of Inclusive Education, 12(5-6), 639-650.

OMS (2011). Informe Mundial sobre la Discapacidad. Ediciones de la OMS. Recuperado de http://www.who.int/disabilities/world_report/2011/es/

Osca-Lluch, J., Civera, C., y Tortosa, F. (2012). Autoría y colaboración científica en la revista ansiedad y estrés. Ansiedad y Estrés, 18(1), 69-78.

Ovejero, A. (1998). Las Relaciones Humanas. Psicología social teórica y aplicada. Madrid: Biblioteca Nueva.

Parasuram, K. (2006).Variables that affect teachers' attitudes towards disability and inclusive education in Mumbai, India. Disability \& Society, 21(3), 231-242.

Piel, S.J. (2007). Introduction: The social position of pupils with special needs in regular education. European Journal of Special Needs Education, 22(1), 1-6.

Piercy, M., Wilton, K., y Townsend, M. (2002). Promoting the Social Acceptance of Young Children with Moderate-Severe Intellectual Disabilities Using Cooperative-Learning Techniques. American Journal on Mental Retardation, 107(5), 352-360. 
Quevedo, R., y López-López, W. (2010). Análisis Bibliométrico de las Revistas Multidisciplinares de Psicología Recientemente Incorporadas en la Web of Science (2008-2009). Psicología: Reflexão e Crítica, 23, 384-408.

Quiles, M.J., Ortigosa, S., Pedroche, S., y Méndez, X. (2000). Investigaciones Psicológicas sobre el cuidado del niño hospitalizado: un análisis bibliométrico. Cuadernos de Medicina Psicosomática y Psiquiatría de Enlace, 53, 27-42.

Rillota, F., y Nettelbeck, T. (2007). Effects of an awareness program on attitudes of students without an intellectual disability towards persons with an intellectual disability. Journal of Intellectual \& Developmental Disability, 32(1), 19-27.

Rizzo, T.L., y Vispoel, W.P. (1991). Physical educators' attributes ant attitudes toward teachering students with handicaps. Adapted Physical Activity Quarterly, 8(1), 4-11.

Rogers, B. (1987). A comparative study of the attitudes of regular education personnel toward mainstreaming students and variables affecting attitudes. Acapulco: Pan American Conference on Rehabilitation and Special Education.

Shelvin, M., y O'Moore, A.M. (2000). Foresting positive attitudes: reactions of mainstream pupils to contact with their counterparts who have severe/profound intellectual disabilities. European Journal of Special Needs Education, 15(2), 206-217.

Slininger, D., Sherrill, C., y Jankowski, C.M. (2000). Children's attitudes toward peers with severe disabilities: Revisiting contact theory. Adapted Physical Activity Quarterly, 17(2), 176-196.

Soodak, L.C., Podell, D.M., y Lehman, L.R. (1998). Teacher, Student, and School Attributes as Predictors of Teachers' Responses to Inclusion. The Journal of Special Education, 31(4), 480-497.

Stephens, D., Stephens, R., y von Eisenhart-Rothe, A. (2000). Attitudes toward hearingimpaired children in less developed countries: A pilot study. Audiology, 39(4), 184-191.

Suriá, R. (2011). Análisis comparativo sobre las actitudes de los estudiantes hacia sus compañeros con discapacidad. Electronic Journal of Research in Educational Psychology, 9(1), 197-216.

Verdugo, M.A., Arias, B., y Jenaro, C. (1994). Actitudes hacia las personas con minusvalía. Madrid: INSERSO.

Verdugo, M.A., Jenaro, C., y Arias, B. (1995). Actitudes sociales y profesionales hacia las personas con discapacidad: estrategias de evaluación e intervención. En M.A. Verdugo (Ed.), Personas con discapacidad. Perspectivas psicopedagógicas y rehabilitadoras (pp. 79-143). Madrid: Siglo XXI de España Editores, S.A. 
Vignes, C., Godeau, E., Sentenac, M., Coley, N., Navarro, F., Grandjean, H., y Arnaud, C. (2009). Determinants of students' attitudes towards peers with disabilities. Developmental Medicine and Child Neurology, 51(6), 473-479.

Woolfson, L. M., y Brady, K. (2009). An investigation of factors impacting on mainstream teachers' beliefs about teaching students with learning difficulties. Educational Psychology, 29(2), 221-238. 
García-Fernández, J.M. et al.

\section{[Página en blanco por razones de paginación]}

